\title{
Toxicity and survival outcomes of adjuvant chemoradiation for gastric and gastroesophageal junction cancer patients treated in period 2006-2009: an institutional experience
}

\author{
M. SLAVIK ${ }^{1}$, P. BURKON ${ }^{1}$, T. KAZDA ${ }^{1,2, \star}$, M. BUDIKOVA ${ }^{3}$, M. SLAVIKOVA ${ }^{1}$, E. DVORAKOVA ${ }^{1}$, P. POSPISIL ${ }^{1}$, J. GOMBOSOVA ${ }^{1}$, P. NAVRATILOVA ${ }^{1}$, \\ P. SLAMPA ${ }^{1,4}$
}

${ }^{1}$ Department of Radiation Oncology, Faculty of Medicine, Masaryk University and Masaryk Memorial Cancer Institute, Brno; ${ }^{2}$ International Clinical Research Center, St. Anne's University Hospital Brno, Czech Republic; ${ }^{3}$ Department of Mathematics and Statistics, Faculty of Science, Masaryk University, Brno; ${ }^{4}$ Regional Centre for Applied Molecular Oncology, Masaryk Memorial Cancer Institute, Brno, Czech Republic

${ }^{*}$ Correspondence: tomas.kazda@mou.cz

Received February 7, 2014 / Accepted April 2, 2014

\begin{abstract}
Surgical resection is the mainstay of gastric or gastroesophageal junction cancer treatment and has curative potential for patients with early-stage disease. In order to improve the poor survival rates, there are two complementary treatment strategies used at most - perioperative chemotherapy based on UK Magic trial or adjuvant chemoradiation based on INT-0116 trial. Daily treatment decision making should be led also by institutional experiences with toxicity evaluation. We evaluated survival and toxicity outcomes of 47 consecutive patients who underwent adjuvant chemoradiation in our institution in the years 2006-2009. 45Gy in 5 weeks with concurrent two cycles of FUFA Mayo regimen chemotherapy were administrated as part of combined treatment. The acute toxicity was relatively mild (CTCAE scale): grade 2 nausea in 26\%, vomiting in 13\%, and diarrhoea grade 1 in $15 \%$ and general abdominal discomfort in $57 \%$ of patients. Grade 3 haematological and infectious complications in $6 \%$ and $2 \%$ respectively. Late adverse events were as follows: grade 1 esophageal toxicity in $17 \%$, signs of mild chronic esophageal ulceration and esophageal stenosis in $9 \%$ of patients (50\% of them had tracheoesophageal fistula). The Kaplan- Meier estimate of the median overall survival was 30.5 months with median 25.7 months disease free survival. The overall survival was statistically significantly affected by the amount of removed positive lymph nodes. For the proper evaluation of radiotherapy role in multimodal treatment approach, results of other clinical trials investigating role of concurrent radiotherapy in administration of perioperative chemotherapy will be necessary. Meanwhile, two equally approaches are possible, all having their pros and cons. Institutional toxicity evaluation is recommended in order to provide the best care possible.
\end{abstract}

Key words: adjuvant chemoradiation, gastric cancer, early toxicity, late toxicity, survival outcomes

Gastric cancer is one of the most common types of tumors and remains the second leading cause of death worldwide [1]. However, incidence in Czech Republic is continuously decreasing from about 27 per 100000 in 1980 to 15.1 per 100 000 in 2010 [2]. Mortality follows this trend. On the other hand, the proportion of disease stage is during last 15 years same despite of well-known risk factors and improvements in diagnostic methodology [3]. Barrett's esophagus and dysplasia

Abbreviations: CTCAE: Common Terminology Criteria for Adverse Events; GEJ: gastroesophageal junction; FUFA: 5-fluorouracil/ folinic acid; RT: radiotherapy; OS: overall survival; DFS: disease free survival are associated with the development of esophageal adenocarcinoma while helicobacter pylori infection, atrophic gastritis, intestinal dysplasia or even metaplasia is related to gastric adenocarcinoma $[4,5]$.

Surgical resection is the mainstay of treatment and has curative potential for patients with early-stage cancer. However, the survival rate of patients with advanced resectable gastric or gastroesophageal junction (GEJ) cancers remains poor despite multidisciplinary treatment strategies, such as perioperative chemotherapy or adjuvant chemoradiation [6].

The largest trial which evaluated the role of chemoradiation in adjuvant setting was the U.S. Intergroup 0116 trial (INT- 
Table 1. Patient and tumor basic characteristics; $\mathrm{T}$ and $\mathrm{N}$ stage: tumor and lymph nodal stage; N/A: not assessed

\begin{tabular}{lc}
\hline Characteristic & Number of patients $(\mathrm{n}=47)$ \\
\hline Sex & \\
male & $35(74 \%)$ \\
female & $12(26 \%)$ \\
Age & \\
median & 60.2 years \\
range & $41.1-74.9$ \\
T stage & \\
T1 & $2(4 \%)$ \\
T2 & $18(38 \%)$ \\
T3 & $25(53 \%)$ \\
T4 & $2(4 \%)$ \\
N stage & \\
N0 & $6(13 \%)$ \\
N1 & $27(57 \%)$ \\
N2 & $7(15 \%)$ \\
N3 & $7(15 \%)$ \\
Grade and histology & \\
G N/A & $5(11 \%)$ \\
G2 & $8(17 \%)$ \\
G3 & $34(72 \%)$ \\
Diffuse type & $14(30 \%)$ \\
Surgery type & \\
R0 & $39(83 \%)$ \\
R1 & $8(17 \%)$ \\
D1 & $24(51 \%)$ \\
D2 & $23(49 \%)$ \\
\hline
\end{tabular}

0116), which have a significant impact on the management of this tumor [7]. The updated results of this INT-0116 trial have shown an improvement in disease free (DFS) and overall survival (OS) after chemoradiation, with acceptable toxicity [8]. Based on these results, many institutions implemented a treatment strategy from INT-0116 into their protocols. On the other hand, the following UK Magic trial, investigating perioperative chemotherapy, reported comparable OS [9]. Ongoing Dutch CRITICS trial has also potential to change practice in terms of omission of adjuvant radiotherapy (RT) [10].

Recently, the indication for adjuvant RT remains the standard of care in case of skipped neoadjuvant chemotherapy. Information about unambiguous RT toxicity institutional profile is useful in physician's daily treatment decision, especially when many different treatment approaches are possible. We retrospectively evaluated outcomes of patients who underwent adjuvant chemoradiation according to INT -0116 protocol in our institution in the years 2006-2009.

\section{Patients and methods}

Patients. During the period from January 2006 to December 2009, a total number of 84 consecutive patients with gastric adenocarcinoma or adenocarcinoma of GEJ were referred to a multidisciplinary gastrointestinal tumor board. 47 (56\%) patients had recommended surgery and subsequent adjuvant treatment in Masaryk Memorial Cancer Institute, Brno, Czech Republic. These patients were enrolled in our retrospective analysis. Resectability and operability were assessed by experienced surgeon and type of operation was at the surgeon's discretion. Basic tumor and patient characteristics are summarized in Table 1. Clinical stage was defined in accordance with AJCC classification, $7^{\text {th }}$ edition, based on postoperative findings. There were 2 stage I patients, 29 with stage II and 16 patients with stage III. All patients underwent adjuvant concurrent chemoradiation after the curative surgical resection of primary cancer and adjacent lymph nodes. Types of surgical procedures varied among these patients according to the extent of local involvement and also depended on a different practice in several institutions where surgery was performed (D1or D2 dissection). A total number of removed lymph nodes and surgical margins were assessed as follows: R0 - clean margins, R1- microscopic involvement or R2macroscopic residual mass (no case). According to histology, there were only adenocarcinomas of the stomach or GEJ in our data set either grade 2 or 3 in all 47 cases (including diffuse histology type in 14 cases). Mutational status of BRAF or KRAS, alterations of E-cadherin gene, MSI or other molecular markers were not systematically examined in our cohort. These tests were not generally available in our institution for gastric cancer patients at time of workup of patients included in this retrospective study.

Chemoradiation and follow-up. Written informed consent was mandatory before starting the treatment. All of the patients were intended to be treated according to our standard protocol for adjuvant therapy which consists of five courses of chemotherapy based on standard 5-fluorouracil/ folinic acid (FUFA Mayo) regimen, with at least two of the cycles (at most second and third course) with concurrent RT. The dosage of chemotherapy was $425 \mathrm{mg} / \mathrm{m}^{2}$ of $5-\mathrm{FU}$ and $20 \mathrm{mg} / \mathrm{m}^{2}$ of folinic acid day 1-5, every 28 days. 31 patients (66\%) underwent all 5 planned chemotherapy cycles; four cycles were applied in 3 cases and 3 cycles in 8 cases. Two patients received 2 cycles and 3 only one cycle of chemotherapy. Radiotherapy was performed in all patients in accordance with our institutional protocol based on irradiation of gastroesophageal anastomosis, gastric and duodenal stump, the tumor bed, and the perigastric nodes on the greater and lesser curvature, coeliac, local para-aortic, splenic, hepato-duodenal and pancreaticoduodenal lymph nodes regions. Target volumes were further adjusted for tumors of the cardia or GEJ (inclusion of lower para-esophageal nodes and omission of pancreaticoduodenal nodes) and for antral tumors with omission of the splenic nodes. These clinical target volumes were subsequently isotropically expanded by $10 \mathrm{~mm}$ creating planning target volumes to accommodate random setup uncertainty. The total dose of $45 \mathrm{~Gy}$ in 25 daily fractions (1.8Gy per fraction) was delivered in 5 weeks. Anterior and posterior opposing 
beams were used in 11 cases and 3D conformal techniques using 3-7coplanar beams in 36 cases. Complete 25 fractions were applied in 37 patients, 9 patients underwent 22 to 24 fractions and one patient got only 20 fractions due to toxicity concerns.

Patients were followed up according to our institutional policy since enrolled patients were outside the frame of clinical trial. History and physical examination were performed every 3 months, abdominal CT scans and gastrofibroscopy at least every 6 months and chest X-ray once a year. After 3 years, CT and gastrofibroscopy were performed once a year and physical examination every 6 months. Other examinations were performed anytime if clinically indicated.

The acute toxicity of combined treatment was determined using Common Terminology Criteria for Adverse Events (CTCAE) v4.0 toxicity criteria [11]. Evaluation of late effects was based on Radiation Therapy Oncology Group (RTOG) toxicity criteria. Survival outcomes were evaluated as OS and progression free survival defined as a time from surgery to the death and to the first signs of relapsed disease respectively. Patterns of relapse were described separately as local - at the anastomosis, regional - in regional lymph node stations and distant - metastatic disease outside the regional lymph node area.

Statistical analysis. The statistical analysis was performed by the Department of Mathematics and Statistics of the Faculty of Science, Masaryk University in Brno, Czech Republic using standardized statistical methods conducted with Statistica system, ver. 12. (StatSoft, Inc.2013). A p value $\leq .05$ was considered to be significant. Pearson's chi-squared test was used for testing the relation between the presence and severity of nausea or vomiting and the RT technique, while Fisher's exact test for the relationship between RT technique and other different acute toxicity symptoms such as diarrhoea, pain, lack of appetite and abdominal discomfort. Kaplan-Meier and Gehan-Wilcoxon test estimate OS and DFS with comparison of survival distribution between defined groups.

\section{Results}

For all 47 patients included in this retrospective study, we reviewed acute gastrointestinal toxicity -nausea, vomiting, diarrhoea, weight loss, pain, abdominal discomfort, haematological toxicity and infectious complications (Table 2). Generally, the acute toxicity profile of concomitant treatment was relatively favourable. Nevertheless, there has been noted one treatment-related death 3 weeks after the end of combined treatment. It was in a case of 74 years old patient with multiple comorbidities and history of metachronous neoplastic triplicity. The reason of death was relapse of pulmonary embolism together with paralytic ileus.

The most common chemoradiation toxicity was related to gastrointestinal tract as well as haematopoiesis (CTCAE scale). In our patients, there were 15 cases (32\%) of nausea grade 1 and 12 cases (25\%) of grade 2, vomiting grade 1 and 2 was noted in $10(21 \%)$ and $6(13 \%)$ cases respectively. Diarrhoea occurred only in 7 (15\%) cases (grade1). Grade 1 pain was reported in 14 (30\%), overall abdominal discomfort in $27(57 \%)$ patients. Weight loss grade 1 was presented in 11 patients (23\%), grade 2 in 2 patients (4\%) and grade 3 in 1 case (2\%). Overall haematological complications were recorded in 18 cases - in 9 patients (19\%) of grade 1 , in 6 patients (13\%) of grade 2 and 3 patients (6\%) developed grade 3 toxicity. This is mixed haematological toxicity- we recorded only the worst grade of either neutropenia or anaemia. Actually, all of grade 3 cases were those of neutropenia. Infectious complications were noted in 3 patients - in 2 patients (4\%) of grade 1 and in one patient (2\%) of grade 2 . In summary, the overall acute toxicity was relatively mild, with only 4 cases of grade 3 CTCAE scale ( 3 cases of haematological toxicity, 1 case of grade 3 weight loss). Further we analysed any possible relationships between RT technique and acute toxicity profile. No statistically significant relationship was found between the presence and severity of nausea or vomiting and the RT technique (p- value 0.302 and 0.854 respectively, chi-squared test).

We also assessed the relationship between RT technique and other acute toxicity symptoms. Testing these variables by Fisher's exact test, the results didn't reach a statistical significance with p-values 0.464 (pain), 0.659 (diarrhoea), 0.638 (abdominal discomfort). As weight loss influences indication as well as fluent course of chemoradiation, we matched previously listed variables with the degree of losing weight. We found out that there was a relationship between the weight

Table 2. Acute toxicity profile $(n=47)$

\begin{tabular}{lcccccccc}
\hline \multirow{2}{*}{ Adverse sequelae } & \multicolumn{2}{c}{ Grade 0 } & \multicolumn{2}{c}{ Grade 1 } & \multicolumn{2}{c}{ Grade 2 } & \multicolumn{2}{c}{ Grade 3 } \\
\cline { 2 - 9 } & $\mathrm{N}$ & $\%$ & $\mathrm{~N}$ & $\%$ & $\mathrm{~N}$ & $\%$ & $\mathrm{~N}$ & $\%$ \\
\hline Nausea & 20 & 43.0 & 15 & 32.0 & 12 & 25.0 & 0 & 0.0 \\
Vomiting & 31 & 66.0 & 10 & 21.0 & 6 & 13.0 & 0 & 0.0 \\
Diarrhea & 40 & 85.0 & 7 & 15.0 & 0 & 0.0 & 0 & 0.0 \\
Weight loss & 33 & 47.7 & 11 & 23.0 & 2 & 4.0 & 1 & 2.0 \\
Abdominal discomfort & 20 & 43.0 & 27 & 57.0 & 0 & 0.0 & 0 & 0.0 \\
Pain & 33 & 70.0 & 14 & 30.0 & 0 & 0.0 & 0 & 0.0 \\
Hematological toxicity & 29 & 62.0 & 9 & 19.0 & 6 & 13.0 & 3 & 6.0 \\
Infectious complication & 44 & 94.0 & 2 & 4.0 & 1 & 2.0 & 0 & 0.0 \\
\hline
\end{tabular}




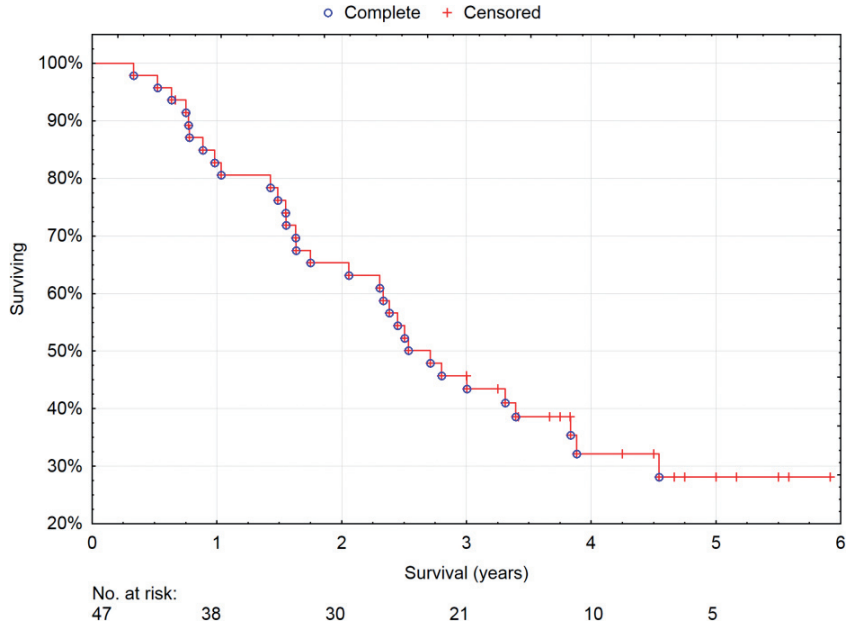

Figure 1. Overall survival among all eligible patients. The median duration of overall survival was $\mathbf{3 0 . 5}$ months based on Kaplan-Meier estimation.

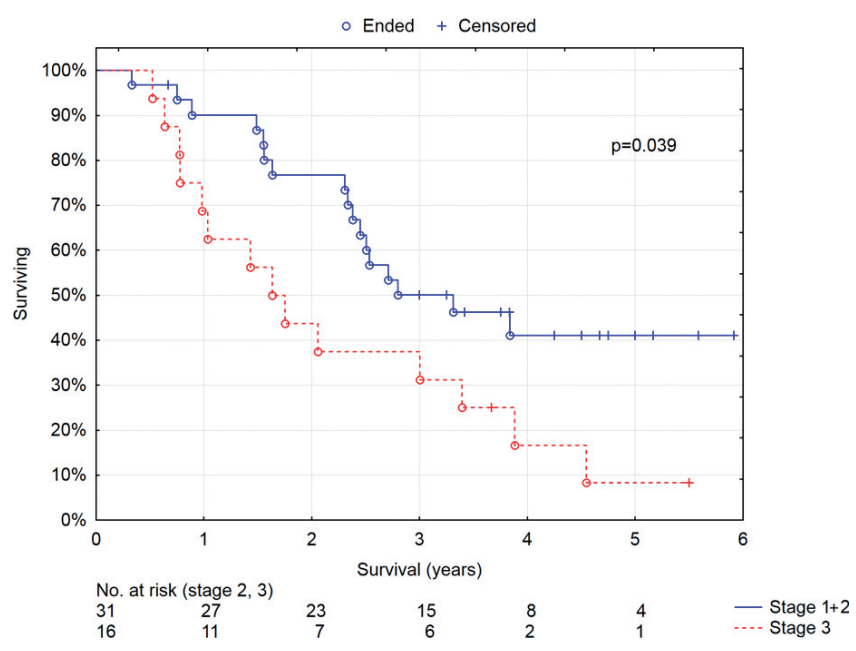

Figure 2. Overall survival among all eligible patients, according to disease stage based on AJCC classification. The median survival in the first group (blue line, 2 stage 1 patients +29 stage 2 patients) was 32.5 months and in stage III patients 19.6 months based on Kaplan-Meier estimation. The difference in overall survival was statistically significant $(p=0.039$, GehanWilcoxon test).

loss and presence and severity of some toxicity related symptoms. There was a proved statistically significant relationship between the weight loss and presence and severity of nausea ( $\mathrm{p}=0.004$, chi-squared test), as well as between weight loss and pain ( $\mathrm{p}=0.014$, Fisher's exact test).

Apart from the acute toxicity symptoms we evaluated also the late toxicity of combined treatment. Because of the retrospective study design, we evaluated only the esophageal toxicity in terms of late esophageal stenosis, ulceration and fistulation. In our cohort we recorded 8 cases $(17 \%)$ of grade 1 esophageal toxicity - slight difficulties with swallowing solid

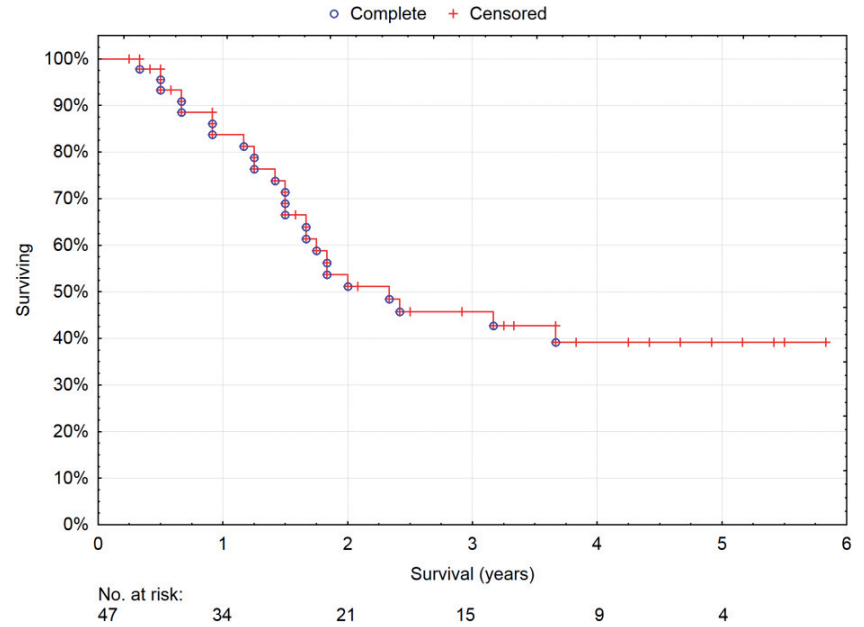

Figure 3. Disease free survival among all eligible patients. The median duration of disease free survival was 25.7 months based on Kaplan-Meier estimation.

food or mild pain during swallowing due to the mild fibrosis without need of any medications or interventions. Signs of mild chronic esophageal ulceration were recorded in 4 cases (9\%). In same amount of patients, esophageal stenosis (one of them with necessity of repeated dilatations) was recorded, and 2 of them had also tracheoesophageal fistula. All of these 4 patient with severe late toxicity had also at least one sign of serious acute gastrointestinal toxicity grade 2 during the course of radiotherapy- all of them had nausea, vomiting and weight loss and abdominal discomfort. No difference in the toxicity (acute as well as late) profile was observed between patients who underwent D1 or D2 surgery (chi-squared or Fisher's exact test).

Overall survival was defined as a period from the time of diagnosis till death and DFS till the first radiographic signs of relapse. With more than 5 years of follow up, the Kaplan- Meier estimate of median OS was 30.5 months regardless on disease stage (Figure 1). Grouping according to the stage, there was a statistically significant difference in OS between stage $1+2$ vs. stage 3 (32.5 vs. 19.6 month, p-value 0.039) (Figure 2). Overall survival was longer in group of patients with D2 surgery (36 months) comparing to D1 (29 months) but these results did not reach statistical significance.

The disease free survival was estimated to be 25.7 months (Figure 3). The patterns of relapse were as follows: there were noted a total number of 24 relapses (51\% patients), thereof 23 were distal recurrences and only 1 isolated relapse at the anastomosis was found. Among the patients with distant recurrences, 10 of them suffered also from local relapse at the site of anastomosis and regional lymph nodes.

As a possible confounders, we tested different variables to have an influence on OS and on either loco-regional or distant recurrence. We matched the total amount of lymph 
nodes removed, total number of positive nodes removed and the quality of surgery (in accordance with R0 or R1 resection) respectively. The only parameter which significantly affected OS was the amount of positive lymph nodes removed- the less number of positive nodes was found in biopsy specimen the better overall survival. As reported in plotted graph (Figure 4), the parameter of OS and the number of positive lymph nodes removed correlate with Pearson's correlation coefficient -0.439 which is significant at the 0.05 significance level. We also tested the relations between the amount of removed nodes (Table 3 ) or positive removed nodes (Table 4) and the presence of local or distant recurrences using Wilcoxon two sample test. The only statistically significant relevance was observed between the number of positive lymph nodes and the amount of distal recurrences.

Assessing extent of surgery, there was no significant difference between groups with R0 and R1 resections and the patterns of relapse. Local relapse at the anastomosis occurred in 10 patients ( 3 of whom had reported R1 resection). Of the 37 patients without local failure, 5 had R1 resection. No statistical difference was found between those groups (Fisher's exact test).

\section{Discussion}

There are obvious differences in the treatment outcomes of gastric cancer across the world. Whilst in Japan were excellent treatment results reached with single modality of D2 dissection with actually better results than those of combined modality approach in western countries, the usefulness of D2 over D1 lymph node dissection in western part of the world is still under discussion and its possible benefit was proved quite recently $[12,13]$. These differences reflect a different epidemiology and perhaps a different etiology of gastric cancer around the world [14]. Instead of these contrasts, it is clear that surgery remains a mainstay of the gastric cancer treatment.

In the western world, there are two common acceptable treatment strategies in resectable gastric cancer- either adjuvant chemoradiation based on INT 0116 trial results or perioperative chemotherapy based on Magic trial with comparable results. D2 resection is now also recommended with trend toward to better OS and less local recurrences $[12,13]$. In case of $\mathrm{D} 2$ resection, there is still an uncertanity in the use of adjuvant RT as a part of combined treatment. Dutch retrospective study showed no significant difference in recurrence rates after D2 dissection [10]. The only prospective Artist trial showed no significant impact on DFS when adding RT to postoperative CHT after D2 resection, but there was statistically significant trend during a subgroup analysis toward to better DFS in node positive group of patients [15]. On the other hand, trials using adjuvant chemotherapy after $\mathrm{D} 2$ resection showed promising results in terms of DFS and OS and demonstrated this approach as feasible $[16,17]$. Majority of these prospective trials were conducted in the eastern part of the world and it

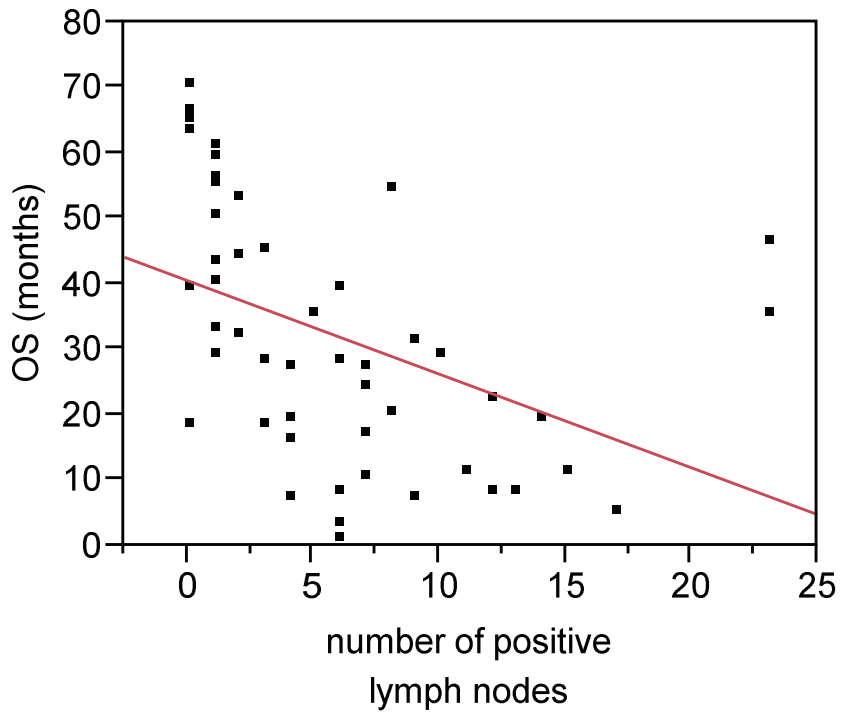

Figure 4. Pearson's correlation of overall survival and number of positive lymph nodes found in resected tissue $\left(p=0.0029^{\star}\right)$ :

is known for gastric cancer to be difficult to generalize these results. A large metaanalysis showed survival benefit when adding RT as a part of a treatment [18].

The overall 5-year survival rate for resected gastric patients in western countries remains poor. The results of a large intergroup American study (INT 0116) reported an improvement of DFS and OS with chemoradiation, which led to the regimen being adopted by many centres [8]. Also our institution has initially implemented the treatment strategy of INT 0116. More recently, the MAGIC/UK Medical Research Council

Table 3. Number of lymph nodes removed correlated to local and distal recurrences

\begin{tabular}{lccc}
\hline Pattern of failure & N & $\begin{array}{c}\text { No of lymphonodes } \\
\text { removed (median) }\end{array}$ & $\begin{array}{c}\text { Wilcoxon two sample } \\
\text { test correlation }\end{array}$ \\
\hline Distant recurrence + & 20 & 12,5 & $\mathrm{p}=0.519$ \\
Distant recurrence - & 22 & 11 & $\mathrm{p}=0.223$ \\
Local recurrence + & 7 & 14 & \\
Local recurrence - & 35 & 11 & \\
\hline
\end{tabular}

Table 4. Number of positive lymph nodes removed correlated to local and distal recurrences

\begin{tabular}{lccc}
\hline Pattern of failure & N & $\begin{array}{c}\text { No of positive } \\
\text { lymphonodes } \\
\text { removed (median) }\end{array}$ & $\begin{array}{c}\text { Wilcoxon two } \\
\text { sample test correlation }\end{array}$ \\
\hline Distant recurrence + & 21 & 6 & $\mathrm{p}=0.023^{*}$ \\
Distant recurrence - & 23 & 1 & $\mathrm{p}=0.209$ \\
Local recurrence + & 8 & 5 & \\
Local recurrence - & 36 & 3.5 & \\
\hline
\end{tabular}


trial demonstrated that perioperative chemotherapy showed comparable OS and progression free survival with INT 0116, so in the last years a lot of physicians tended to use perioperative chemotherapy according to the Magic trial [9].

As a part of this modification, we tended to collect our own data on treatment of gastric cancer patients who underwent a treatment based on INT 0116 protocol. This brings several questions to be discussed. First of all is the quality of surgery. In our group, there were 8 cases of $\mathrm{R} 1$ resections and the minimum 15 lymph nodes dissected was reached in only 12 cases with overall median of 12 lymph nodes dissected (2-24 nodes). The relatively large number of $\mathrm{R} 1$ resections is probably due to the fact, that not all the surgical procedures took place in our institution or another major institution with well-trained team of gastric surgeons. Instead of this, there is a lot of R1 dissections reported in the other past evaluations $[19,20,21]$. It can be concluded that it is at much importance for gastric cancer patients to undergo a surgical procedure on well situated place with trained personnel, because surgery is the mainstay of gastric cancer treatment, and D2 dissection is achievable especially by well-trained surgery team [12].

The overall acute toxicity of combined treatment in our series was relatively mild, with maximum of 4 cases of grade 3 in CTCAE scale. But it must be regarded with all the awareness according to the retrospective dataset with all its limitations and inaccuracy, and with respect to that not all of the patients underwent the entire planned treatment- due to the toxicity concerns related to age or weight loss, only 31 of all 47 patients (66\%) underwent 5 cycles of adjuvant chemotherapy. The dose of radiation varied less, but $45.0 \mathrm{~Gy}$ received 37 of 47 patients (79\%). The weight loss was proven to have impact on the presence and severity of other adverse events of treatment such as nausea, pain or lack of appetite. Malnutrition is known to be associated with many adverse events during the treatment such as dose-limiting toxicity, more unplanned breaks in the treatment, lower responsiveness to treatment, and lower quality of life $[22,23,24]$. Regardless of chosen strategy - perioperative chemotherapy or adjuvant combined treatment - the much effort should be paid on nutritional status of these patients, before, during and after completed therapy to avoid a vicious circle between treatment related toxicities, inadequate food intake and malnutrition.

The long term toxicity in our group of patient was quite favourable. Severe late toxicity like esophageal stenosis was recorded in 4 cases (9\%) - it is notable that all 4 patients with stenosis had also considerable acute gastrointestinal toxicity. So it might be related to consequentional late effects of multimodal therapy. Combined cause of treatment toxicity is also supported by the fact, that there is no differentiation in toxicity outcomes relating the RT technique (anterior and posterior opposing beams versus 3 -dimensional conformal techniques using 3-7 coplanar beams).

Our cohort is still too small to make any implications for OS based on the analysis of different variables such as total amount of lymph nodes removed, total number of positive nodes removed and the quality of surgery. Apart from low volume setting of our group, the median number of evaluated lymph nodes is too small to debate any stage migration effect. There was no statistically significant relationship between the number of lymph nodes resected and the OS or between the number of nodes removed and the local or distant sites of relapse. The only relevant was the number of positive lymph nodes found in the surgical specimen in our group which negatively correlated with OS and number of distant relapse; which probably reflects simply a larger burden of disease at the time of diagnosis resulting in worse outcomes and is in accordance with another observation $[25,26]$.

The overall survival in our group is in some aspects comparable with results of another retrospective series from the western world centres (Table 5). DFS in our study was 27.5 months. In INT 0116 was 19 months in the surgery-only group and 30 months in the chemoradiotherapy group [8]. Direct comparison with a key trial INT 0116 is however not possible because of the huge differences of these series such as retrospective design of our study, number of patients and different proportion of R1 resections. On the other hand these results reflect the common situation of treating gastric cancer patients under conditions outside of well designed clinical trials.

In summary, adjuvant chemoradiation is still one of equivalent treatment options for patients with resectable gastric

Table 5. Results of prospective and retrospective trials evaluating adjuvant concurrent chemoradiation for GEJ or gastric adenocarcinomas in western world(if comparative studies, results ofchemoradiation treatment arms only displayed):

\begin{tabular}{|c|c|c|c|c|c|c|c|}
\hline Study & $\mathrm{N}$ & $\begin{array}{l}\text { Dissection } \\
\text { status }\end{array}$ & $\begin{array}{c}\mathrm{F} / \mathrm{U} \\
\text { (months) }\end{array}$ & $\begin{array}{c}2 \text { yrs } \\
\text { survival }\end{array}$ & $\begin{array}{c}3 \text { yrs } \\
\text { survival }\end{array}$ & $\begin{array}{c}5 \text { yrs } \\
\text { survival }\end{array}$ & $\begin{array}{l}\text { Median survival } \\
\text { (months) }\end{array}$ \\
\hline \multicolumn{8}{|l|}{ Prospective } \\
\hline INT 0116 (USA), [7] & 281 & $\mathrm{D} 1 / \mathrm{D} 2$ & 60 & over $60 \%$ & $50 \%$ & $40 \%$ & 35 \\
\hline \multicolumn{8}{|l|}{ Retrospective } \\
\hline Dikken (Holland), [19] & 91 & $\mathrm{D} 1 / \mathrm{D} 2$ & 19 & $71 \%$ & - & - & - \\
\hline Jacomé (Brazil), [27] & 101 & $\mathrm{D} 2$ & 31.6 & - & $64.4 \%$ & - & - \\
\hline Hempel (Poland), [25] & 37 & - & 14 & - & - & - & - \\
\hline Isa (Spain, Chile), [28] & 80 & $\mathrm{D} 1 / \mathrm{D} 2$ & 25.8 & $64.3 \%$ & - & - & - \\
\hline This study (Czech) & 47 & $\mathrm{D} 1 / \mathrm{D} 2$ & 30.4 & 63.8 & 44.6 & - & 30.5 \\
\hline
\end{tabular}


cancer. Individual institutions should evaluate their own toxicity profiles to aid decision making for selection of appropriate treatment of their own patients. For the proper implementation of radiotherapy in multimodal adjuvant approach of resectable gastric cancer, we have to wait for the results of Dutch CRITICS trial investigating perioperative chemotherapy with or without postoperative concurrent radiotherapy.

Acknowledgements: Supported by MH CZ - DRO (MMCI, 00209805). This article was supported by Ministry of Education, Youth and Sports of the CzechRepublic through A-Math-Net Project no. CZ.1.07/2.4.00/17.0100. Supported by European Regional Development Fund - Project FNUSA-ICRC (No.CZ.1.05/1.1.00/02.0123), European Social Fund and the State Budget of the Czech Republic. Supported by European Regional Development Fund and the State Budget of the Czech Republic (RECAMO, CZ.1.05/2.1.00/ 03.0101).

\section{References}

[1] KELLEY JR, DUGGAN JM. Gastric cancer epidemiology and risk factors.J ClinEpidemiol 2003; 56: 1-9. http://dx.doi. org/10.1016/S0895-4356(02)00534-6

[2] DUSEK L, MUZIK J, KUBASEK M, KOPTIKOVA J, ZALOUDIK J et al. Epidemiology of Malignant Tumours in the Czech Republic [online]. Masaryk University, Czech Republic, [2005], [cit. 2014-2-06]. http: //www.svod.cz. Version 7.0 [2007], ISSN $1802-8861$.

[3] DUSEK L, MUZIK J, GELNAROVA E, FINEK J, VYZULA R et al. Cancer incidence and mortality in the Czech Republic. KlinOnkol. 2010; 23: 311-24.

[4] MONTGOMERY E, GOLDBLUM JR GREENSON JK, HABER MM, LAMPS LW et al. Dysplasia as a predictive marker for invasive carcinoma in Barrett esophagus: a follow-up study based on 138 cases from a diagnostic variability study. Hum Pathol 2001; 32: 379-88. http://dx.doi.org/10.1053/ hupa.2001.23511

[5] CORREA P. Helicobacter pylori and gastric cancer: state of the art. Cancer Epidemiol Biomarkers Prev 1996; 5: 477-481.

[6] ASFRAF N, HOFFE S, KIM R. Adjuvant treatment for gastric cancer: chemotherapy versus radiation. Oncologist2013; 18: 1013-21. http://dx.doi.org/10.1634/theoncologist.2012$\underline{0462}$

[7] MACDONALD JS, SMALLEY SR, BENEDETTI J, HUNDAHL SA, ESTES NC et al. Chemoradiotherapy after surgery compared with surgery alone for adenocarcinoma of the stomach or gastroesophageal junction. N Eng J Med 2001; 345: 725-730, http://dx.doi.org/10.1056/NEJMoa010187

[8] SMALLEY SR, BENEDETTI JK, HALLER DG, HUNDAHL SA, ESTES NC et al. Updated analysis of SWOG-directed intergroup study 0116: a phase III trial of adjuvant radiochemotherapy versus observation after curative gastric cancer resection. J ClinOncol. 2012; 30: 2327-33. http://dx.doi. org/10.1200/JCO.2011.36.7136

[9] CUNINGHAM D, ALLUM WH, STENNING SP, THOMPSAON JN, VAN DE VELDE CJ et al. Perioperative chemotherapy versus surgery alone for resectablegastroesopha- geal cancer. New Eng J Med 2006; 355: 11-22. http://dx.doi. org/10.1056/NEJMoa055531

[10] DIKKEN JL, VAN SANDICK JW, MAURITS SWELLENGREBEL HA, LIND PA, PUTTER $\mathrm{H}$ et al. Neo-adjuvant chemotherapy followed by surgery and chemotherapy or by surgery and chemoradiotherapy for patients with resectable gastric cancer (CRITICS). BMC Cancer 2011; 11: 329. http: //www.ncbi.nlm.nih.gov/pmc/articles/PMC3175221/.

[11] National Cancer InstituteCommon Terminology Criteria for Adverse Events v4.0 NCI, NIH, DHHS. May 29, 2009NIH publication \# 09-7473

[12] DEGIULI M, SASAKO M, PONTI A. Morbidity and mortality in the Italian Gastric Cancer Study Group randomized clinical trial of D1 versus D2 resection for gastric cancer. Br J Surg. 2010; 97: 643-9. http://dx.doi.org/10.1002/bjs.6936

[13] SEEVARATNAM R, BOCICARIU A, CARDOSO R, MAHAR A, KISS A et al. A meta-analysis of D1 versus D2 lymph node dissection. Gastric Cancer 2012; 15 (Suppl 1): S60-S69. http:// dx.doi.org/10.1007/s10120-011-0110-9

[14] MACDONALD JS. Gastric cancer: Nagoya is not New York. J ClinOncol. 2011; 29: 4348-50. http://dx.doi.org/10.1200/ LCO.2011.37.5691

[15] LEE J, DO LIM H, KIM S, PARK SH, PARK JO et al. Phase III Trial Comparing Capecitabine Plus Cisplatin Versus Capecitabine Plus Cisplatin With Concurrent Capecitabine Radiotherapy in Completely Resected Gastric Cancer With D2 Lymph Node Dissection: The ARTIST Trial. J ClinOncol.2012; 30: 268-273. http://dx.doi.org/10.1200/JCO.2011.39.1953

[16] SAKARUMOTO S, SASAKOM, YAMAGUCHI T, KINOSHITA T, FUJII M et al. Adjuvant chemotherapy for gastric cancer with S-1, an oral fluoropyrimidine. N Engl J Med. 2007; 357: 1810-20. http://dx.doi.org/10.1056/NEJMoa072252

[17] BANG YJ, KIM YW, YANG HK, CHUNG HC, PARK YK et al. Adjuvant capecitabine and oxaliplatin for gastric cancer after D2 gastrectomy (CLASSIC): a phase 3 open-label, randomised controlled trial. Lancet. 2012; 379: 315-21. http://dx.doi. org/10.1016/S0140-6736(11)61873-4

[18] VALENTINI V, CELLINI F, MINSKY BD, MATTIUCCI GC, BALDUCCI $\mathrm{M}$ et al. Survival after radiotherapy in gastric cancer: Systematic review and meta-analysis. Radiotherapy and Oncology 2009; 92: 176-183. http://dx.doi.org/10.1016/ j.radonc.2009.06.014

[19] DIKKEN JL, JANSEN E, CATS A, BAKKER B, HARTGRINK $\mathrm{HH}$ et al. Impact of the Extent of Surgery and Postoperative Chemoradiotherapy on Recurrence Patterns in Gastric Cancer. J ClinOncol. 2010 May 10; 28: 2430-6. http://dx.doi. org/10.1200/JCO.2009.26.9654

[20] SIEWERT JR, BOTTCHER K, STEIN HJ, RODER JD. Relevant prognostic factors in gastric cancer: ten year results of the German Gastric Cancer Study. Ann Surg 1998; 228: 449-61. http://dx.doi.org/10.1097/00000658-199810000-00002

[21] HALLISSEY MT, JEWKES AJ, DUNN JA, FIELDING JW. Resection-line involvement in gastric cancer: a continuing problem. Br J Surg 1993; 80: 418-20. http://dx.doi.org/ 10.1002/bjs.1800801121

[22] ANDREYEV HJ, NORMAN AR, OATES J, CUNNINGHAM D. Why do patients with weight loss have a worse outcome 
when undergoing chemotherapy for gastrointestinal malignancies? Eur J Cancer. 1998; 34: 503-9. http://dx.doi. org/10.1016/S0959-8049(97)10090-9

[23] HILl A, KISS N, HODGSON B, CROWE TC, WALSH AD. Associations between nutritional status, weight loss, radiotherapy treatment toxicity and treatment outcomes in gastrointestinal cancer patients.ClinNutr. 2011; 30: 92-8. http://dx.doi.org/10.1016/j.clnu.2010.07.015

[24] MIYATA H, YANO M, YASUDA T, HAMANO R, YAMASAKI $M$ et al. Randomized study of clinical effect of enteral nutrition support during neoadjuvant chemotherapy on chemotherapy-related toxicity in patients with esophageal cancer. ClinNutr. 2012; 31:330-6. http://dx.doi.org/10.1016/ j.clnu.2011.11.002

[25] HEMPEL D, POPLAWSKA-MAKSIMOVICZ E, BARBARA PJ, SIERKO E et al. AdiuvantRadiochemotherapy for gastric cancer patients - single-institution retrospective experience. Radiotherapy \& Oncology 2011; 99, Supplement 1: S355. http://dx.doi.org/10.1016/S0167-8140(11)71046-5

[26] WU CW, HSIEH MC, LO SS, TSAY SH, LUI WY et al. Relation of number of positive lymph nodes to the prognosis of patients with primary gastric adenocarcinoma. Gut 1996; 38: 525-527. http://dx.doi.org/10.1136/gut.38.4.525

[27] JACOME AA, WOHNRATH DR, SCAPULATEMPO NETO C, FREGNANI JH, QUINTO AL et al. Effect of adjuvant chemoradiotherapy on overall survival of gastric cancer patients submitted to D2 lymphadenectomy. Gastric Cancer. 2013; 16: 233-8. http://dx.doi.org/10.1007/s10120-012-0171-4

[28] ISA N, SOLIS J. Adjuvant radiochemotherapy in gastric cancer completely resected: Hospital Van Buren experience, Valparaiso, Chile. Radiotherapy \& Oncology 2011; 99, Supplement 1: S355. http://dx.doi.org/10.1016/S0167-8140(11)71047-7 